TRANSACTIONS OF THE

AMERICAN MATHEMATICAL SOCIETY

Volume 348, Number 6, June 1996

\title{
HARDY SPACES AND TWISTED SECTORS FOR GEOMETRIC MODELS
}

\author{
PIETRO POGGI-CORRADINI
}

\begin{abstract}
We study the one-to-one analytic maps $\sigma$ that send the unit disc into a region $G$ with the property that $\lambda G \subset G$ for some complex number $\lambda$, $0<|\lambda|<1$. These functions arise in iteration theory, giving a model for the self-maps of the unit disk into itself, and in the study of composition operators as their eigenfunctions. We show that for such functions there are geometrical conditions on the image region $G$ that characterize their rate of growth, i.e. we prove that $\sigma \in \bigcap_{p<\infty} H^{p}$ if and only if $G$ does not contain a twisted sector. Then, we examine the connection with composition operators, and further investigate the no twisted sector condition. Finally, in the Appendix, we give a different proof of a result of J. Shapiro about the essential norm of a composition operator.
\end{abstract}

\section{INTRODUCTION}

In the following, we will denote by $\operatorname{cl}(E), \operatorname{int}(E)$, and $d(\cdot, E)$, the closure, the interior, and the distance to the set $E$; by $B(z, r)$, the euclidean ball centered at $z$ of radius $r$; and by $\omega(z, J, \Omega)$, the harmonic measure at $z$, of the set $\operatorname{cl}(J) \cap \operatorname{cl}(\Omega)$, relative to the component of the region $\Omega \backslash \operatorname{cl}(J)$ that contains $z$.

1.1. Geometric models. Let $U$ be the unit disk in the complex plane. Consider an analytic function $\sigma: U \longrightarrow G$ that is one-to-one and onto $G$, such that $\sigma(0)=0$ and $\sigma^{\prime}(0)=1$. Moreover, assume that $\lambda G \subset G$, for some complex number $\lambda$ with $0<|\lambda|<1$. This condition arises in the context of geometric models for univalent maps of $U$ into itself; see [9] and [11]. In fact, since $\lambda G \subset G$, we can define $\phi(w)=\sigma^{-1}(\lambda \sigma(w))$ for all $w \in U$. Then $\phi$ is a one-to-one analytic map of $U$ into itself with $\phi(0)=0$ and $\phi^{\prime}(0)=\lambda$. Conversely, given a map $\phi$ of $U$ into itself that is one-to-one and analytic, let $\lambda=\phi^{\prime}(0) \neq 0$ and let $\phi_{n}=\phi \circ \cdots \circ \phi$ be the $n^{t h}$ iterate of $\phi$. Then, $\lambda^{-n} \phi_{n}$ converge uniformly on compact subsets of $U$ to a map $\sigma$ with the following properties: $\sigma$ is univalent on $U, \lambda G \subset G$ with $G=\sigma(U)$, and $\sigma^{-1}(\lambda \sigma)=\phi$. The pair $(G, \lambda)$ is called a model for the map $\phi$. This is used, for instance, in iteration theory: when studying the iterates $\phi_{n}$ of the map $\phi$ it is enough to look at the action of $\lambda^{n} z$ on the set $G$, hence transferring the analytic properties of $\phi_{n}$ into the geometry of $G$. Furthermore, any analytic map $\psi$ of $U$ into itself induces an operator $C_{\psi}$ on the space of analytic functions

Received by the editors November 16, 1994 and, in revised form, June 13, 1995.

1991 Mathematics Subject Classification. Primary 30C45, 30D55, 47B38.

The author acknowledges support from INDAM (Istituto Nazionale di Alta Matematica) while studying at the University of Washington, and wishes to thank Professor D. Marshall for his help and advice. 
$H(U)$, that is defined by precomposition, $C_{\psi}(f)=f \circ \psi$ for all $f \in H(U)$, and is called the composition operator of symbol $\psi$. Therefore, if $\phi$ is a map as above, we can consider the composition operator $C_{\phi}$, where its symbol $\phi$ equals $\sigma^{-1}(\lambda \sigma)$ for some function $\sigma$. In this case, $\sigma$ can be interpreted as an eigenfunction of the linear transformation $C_{\phi}$, because $C_{\phi}(\sigma)=\sigma \circ \phi=\lambda \sigma$. And, from $\sigma$ one can completely determine the spectrum of $C_{\phi}$, since, by Kœnigs Theorem (see [11], p.93), the numbers $\left\{\lambda^{n}\right\}$ are exactly all the eigenvalues of $C_{\phi}$, each has multiplicity one, and the function $\sigma^{n}$ spans the eigenspace for $\lambda^{n}$.

Now, recall that, for $0<p<\infty$, the Hardy spaces are spaces of analytic functions defined as follows:

$$
H^{p}(U)=\left\{f \in H(U): \sup _{0<t<1} \int_{0}^{2 \pi}\left|f\left(t e^{i \theta}\right)\right|^{p} d \theta<\infty\right\} .
$$

Hardy spaces have the property that $H^{p_{1}} \subset H^{p_{2}}$ if $p_{2}<p_{1}$. Moreover, it is well-known that composition operators are bounded operators of the Banach space $H^{p}$ into itself, whenever $1 \leq p<\infty$, because precomposition preserves harmonic majorants. So it is natural to ask when is $\sigma$ an eigenfunction for $C_{\phi}$ acting on $H^{p}$, for $1 \leq p<\infty$, i.e. when is $\sigma \in \bigcap_{p<\infty} H^{p}$ ?

1.2. Twisted sectors. Consider $H^{\infty}$, the space of bounded analytic functions, and $B M O A$, the subspace of $H^{2}$ of functions whose boundary values have bounded mean oscillation. These spaces are related to the Hardy spaces in the following fashion:

$$
H^{\infty} \subset B M O A \subset \bigcap_{p<\infty} H^{p}
$$

Now, a function in $H^{\infty}$ is obviously characterized in terms of its image set $G$ by the fact that $G$ is a bounded set. Pommerenke showed that a similar characterization holds for univalent functions in $B M O A$, see [8] and also [13]:

$$
A \text { univalent map } \sigma \text { is in BMOA if and only if } \sup _{z \in G} d(z, \partial G)<\infty \text {. }
$$

Our goal is to show that there is a similar condition on the set $G$ that allows us to decide when the map $\sigma$ for a geometric model is in $\bigcap_{p<\infty} H^{p}$. In view of Pommerenke's result, we need to study the case when $\sup _{z \in G} d(z, \partial G)=\infty$.

Recall that the map to the right half-plane, $\sigma_{0}=(1+z) /(1-z)$, does not belong to $H^{p}$ for $p \geq 1$. Hence, $\sigma_{0}^{1 / s} \notin H^{p}$ for $p \geq s>1$. The map $\sigma_{0}^{1 / s}$ maps $U$ into an angular sector with angle $\pi / s$. Therefore, by a subordination argument, any map $\sigma$ for which $G=\sigma(U)$ contains an angular sector does not belong to $\bigcap_{p<\infty} H^{p}$. In [9] this condition has been improved by allowing the sector to be twisted. Given a set $E \subset \mathbf{C} \backslash\{0\}$ and $\epsilon>0$, define $S_{\epsilon}[E]=\bigcup_{z \in E} B(z, \epsilon|z|)$. If $\gamma$ is a curve in $\mathbf{C} \backslash\{0\}$, from 0 to $\infty, S_{\epsilon}[\gamma]$ is called a twisted sector. Thus, if $G$ contains a twisted sector $S_{\epsilon}[\gamma]$, we have $d(z, \partial G) \geq \epsilon|z|$, for all $z \in \gamma$. With this new definition at hand, the authors of [9] show, in the proof of Theorem 3.1, that:

$$
\text { If } \sigma \in \bigcap_{p<\infty} H^{p} \text {, then } G \text { cannot contain any twisted sector. }
$$

In general the converse fails, see Section 2. Nevertheless, we will show that the converse holds, when $\sigma$ has the property that $\lambda G \subset G$, for some $\lambda$ such that $0<|\lambda|<1$. Note that, under some further restricting hypothesis, one can obtain the converse from the results of [9]. In fact, suppose that $G$ contains no twisted sectors and that $\lambda G \subset G$. We can form the map $\phi=\sigma^{-1}(\lambda \sigma)$. Then, if for 
some iterate $\phi_{n_{0}}$ of $\phi$ there are only finitely many points on $\partial U$ where $\phi_{n_{0}}$ has an angular derivative and absolute value one, it follows from [9] that $\sigma \in \bigcap_{p<\infty} H^{p}$. This happens, for instance, if $\lambda \partial G \subset G$ and $G$ has only finitely many prime ends at infinity. On the other hand, it also follows from [9] that the converse holds when $G$ is strictly starlike, but this is already clear from Theorem 4.1. of [6], without the condition $\lambda G \subset G$. We remark that these extra conditions assumed in [9] serve a different purpose than ours and that the above implications are obtained from the results of [9] in an indirect way. In the following, we want to show that if $\lambda G \subset G$ and if $G$ contains no twisted sectors, then $\sigma \in \bigcap_{p<\infty} H^{p}$, without assuming any further hypothesis on $G$. To this end, we make the following definition:

Definition 1.1. Given a twisted sector $S_{\epsilon}[\gamma] \subset G$, for some $\epsilon>0$ and some curve $\gamma$ connecting 0 to $\infty$ in $\mathbf{C} \backslash\{0\}$, assume that there is an integer $k \geq 1$ such that $\lambda^{k} \gamma=\gamma$. Then, we say that $G$ contains a $k$-invariant twisted sector.

With this new definition we are able to prove the following theorem.

Theorem 1.2. Suppose $\sigma: U \longrightarrow G$ is a univalent map with $\sigma(0)=0$ and $\sigma^{\prime}(0)=1$, such that $\lambda G \subset G$ for some $0<|\lambda|<1$. Then the following are equivalent:

(a) $G$ does not contain a $k$-invariant twisted sector for any $k \geq 1$.

(b) $G$ does not contain a twisted sector.

(c) $\sigma \in \bigcap_{p<\infty} H^{p}$.

The proof of Theorem 1.2 is given in Section 3. In Section 2 we will describe some of the more relevant examples connected to our problem. Then, in Section 4 we will show how composition operators enter into this subject. Finally, in Section 5 , we explore some further properties that are equivalent to (a), (b) and (c) of Theorem 1.2, and raise a question for future investigations. In the Appendix we give a new proof of J. Shapiro's estimate for the essential norm of a composition operator.

\section{EXAMPLES AND MOTIVATIONS}

2.1. A counterexample. We first show that if we drop the condition that $\lambda G \subset G$ for some $0<|\lambda|<1$, Theorem 1.2 doesn't hold. We will produce a univalent map $\sigma$ on $U$ so that $G=\sigma(U)$ does not contain a twisted sector, yet $\sigma \notin H^{p}$ for some $p<\infty$. This example is due to W. Smith. Let $G$ be the right half-plane minus a sequence of vertical slits $\left\{A_{n}, B_{n}\right\}$. The slit $A_{n}$ is $\{z \in \mathbf{C}: \operatorname{Im}(z) \geq 1 ; \operatorname{Re}(z)=$ $\left.x_{n}\right\}$, with $x_{n}>0$ and $x_{n} \uparrow \infty$, while $B_{n}$ is its reflection about the real line $\mathbf{R}$. Thus $G$ is symmetric about $\mathbf{R}$. Then, no matter how rapidly $x_{n}$ goes to infinity, $G$ contains no twisted sectors. But, if $x_{n}$ goes to infinity rapidly enough, the map $\sigma-x_{n}$ eventually looks like the map to the right half-plane on large portions of $U$. So $\sigma \notin H^{p}$ for some $p<\infty$. More precisely, let $a_{n}$ be the tip of $A_{n}$, i.e. $a_{n}=x_{n}+i$. We can choose $\sigma$ to be real on $\mathbf{R}$ and $\lim _{t \rightarrow 1^{-}} \sigma(t)=+\infty$. Let $\sigma\left(t_{n}\right)=x_{n}$ for $n \geq 0$, and let $t_{0}=0$. If $\rho$ is the hyperbolic metric, using a standard estimate of $\rho$, we obtain:

$$
\rho_{G}\left(x_{0}, x_{n}\right) \leq 2 \int_{x_{0}}^{x_{n}} \frac{1}{d(x, \partial G)} d x \leq 4 \sum_{k=0}^{n-1} \int_{x_{k}}^{x_{k+1}} \frac{1}{\left|x-a_{k}\right|} d x
$$


Note that $\left|x-a_{k}\right|=\sqrt{\left(x-x_{k}\right)^{2}+1} \geq\left|\left(x-x_{k}\right)-1\right|$. So, if $r_{k}=x_{k+1}-x_{k}$,

$$
\int_{x_{k}}^{x_{k+1}} \frac{1}{\left|x-a_{k}\right|} d x \leq \int_{0}^{r_{k}} \frac{1}{|s-1|} d s=\log \left|r_{k}-1\right| \leq \log x_{k+1} .
$$

Therefore, if we set $x_{n}=2^{2^{n}}$,

$$
\rho_{G}\left(x_{0}, x_{n}\right) \leq 4 \sum_{k=0}^{n-1} \log 2^{2^{k+1}}=4 \log 2 \sum_{k=0}^{n-1} 2^{k+1} \leq 8(\log 2) 2^{n}=8 \log x_{n} .
$$

Hence:

$$
\log \frac{1+t_{n}}{1-t_{n}}=\rho_{U}\left(0, t_{n}\right)=\rho_{G}\left(x_{0}, x_{n}\right) \leq 8 \log \sigma\left(t_{n}\right),
$$

i.e. $\sigma\left(t_{n}\right) \geq 1 /\left(1-t_{n}\right)^{1 / 8}$. So, $\sigma \notin H^{p}$, for $p>8$, because each $\sigma \in H^{p}, 0<p<\infty$, obeys the growth restriction $|\sigma(z)| \leq C_{p}\|\sigma\|_{p} /(1-|z|)^{1 / p}$; see [3], §5.5, Thm. 5.9.

2.2. The key example. To show $\sigma \in \bigcap_{p<\infty} H^{p}$ we will estimate the $H^{p}$-norms of $\sigma$ using its distribution function, hence reducing the problem to an estimate of harmonic measure on $G$. More precisely, we will need to estimate the decay of harmonic measure $\omega(0, \partial(\alpha U), G)$, as $\alpha \in \mathbf{R}^{+}$goes to $+\infty$; see Section 3.1 for the details.

Now, in the counter-example of Section 2.1, consider the prime end at infinity of $G$ corresponding to the real line. The property that $G$ contains no twisted sectors tells us that any path to this prime end at infinity must encounter openings of arbitrary small angular size, i.e. the segments $\left(x_{n}+i, x_{n}-i\right)$, but it does not impose restrictions on the frequency of occurrance of these openings. If our region $G$ had the additional property that $\lambda G \subset G$ for some $0<\lambda<1$, given a couple of vertical slits $\left\{A_{N}, B_{N}\right\}$ for some $N$, we would also need the slits $\lambda^{-n} A_{N}$ and $\lambda^{-n} B_{N}$ to be in the complement of $G$ for all $n \geq 0$. Hence, the points $x_{n}$ would have to have the property that $x_{n}=\lambda^{-n} x_{0}$. So, a path to the prime end at infinity corresponding to $\mathbf{R}$ would have to eventually encounter openings of arbitrary small angular size with a given frequency depending on $\lambda$. This suggests trying to estimate $\omega(0, \partial(\alpha U), G)$ using the hyperbolic metric and the geometry of $G$ in terms of small angular openings and the frequency of their occurrence. Unfortunately, problems arise when $G$ has infinitely many prime ends $\left\{E_{n}\right\}$ at infinity such that $\lambda^{k} E_{n} \neq E_{n}$ for all $k>0$ and all $n$. We will see in the next example that in this case the frequency of the small openings can be lost. This is also the example that suggested to us the way to prove Theorem 1.2. Let $G$ be the right half-plane minus infinitely many distinct curves $\left\{\gamma_{n}\right\}_{-\infty}^{+\infty}$ going to infinity; see Figure 1. Namely, consider $x_{0}=1$ and $x_{n}=e^{i \theta_{n}}$, with $\theta_{n}>0$ and $\theta_{n} \uparrow \pi / 2$ in such a way that $\theta_{n}-\theta_{n-1} \downarrow 0$. Also, let $x_{-n}=e^{-i \theta_{n}}$. For $k \geq 0$, consider the sequence of points $2^{k} x_{n+k}$, and let $\gamma_{n}=\bigcup_{k=0}^{+\infty}\left[2^{k} x_{n+k}, 2^{k+1} x_{n+k+1}\right]$ be the polygonal curve that connects them. We set $\lambda=1 / 2$. Then:

$$
\begin{aligned}
2 \gamma_{n} & =\bigcup_{k=0}^{+\infty} 2\left[2^{k} x_{n+k}, 2^{k+1} x_{n+k+1}\right]=\bigcup_{k=0}^{+\infty}\left[2^{k+1} x_{n+k}, 2^{k+2} x_{n+k+1}\right] \\
& =\bigcup_{k=1}^{+\infty}\left[2^{k} x_{(n-1)+k}, 2^{k+1} x_{(n-1)+k+1}\right] \subset \gamma_{n-1} .
\end{aligned}
$$

So $\lambda G \subset G$, for $\lambda=1 / 2$. 


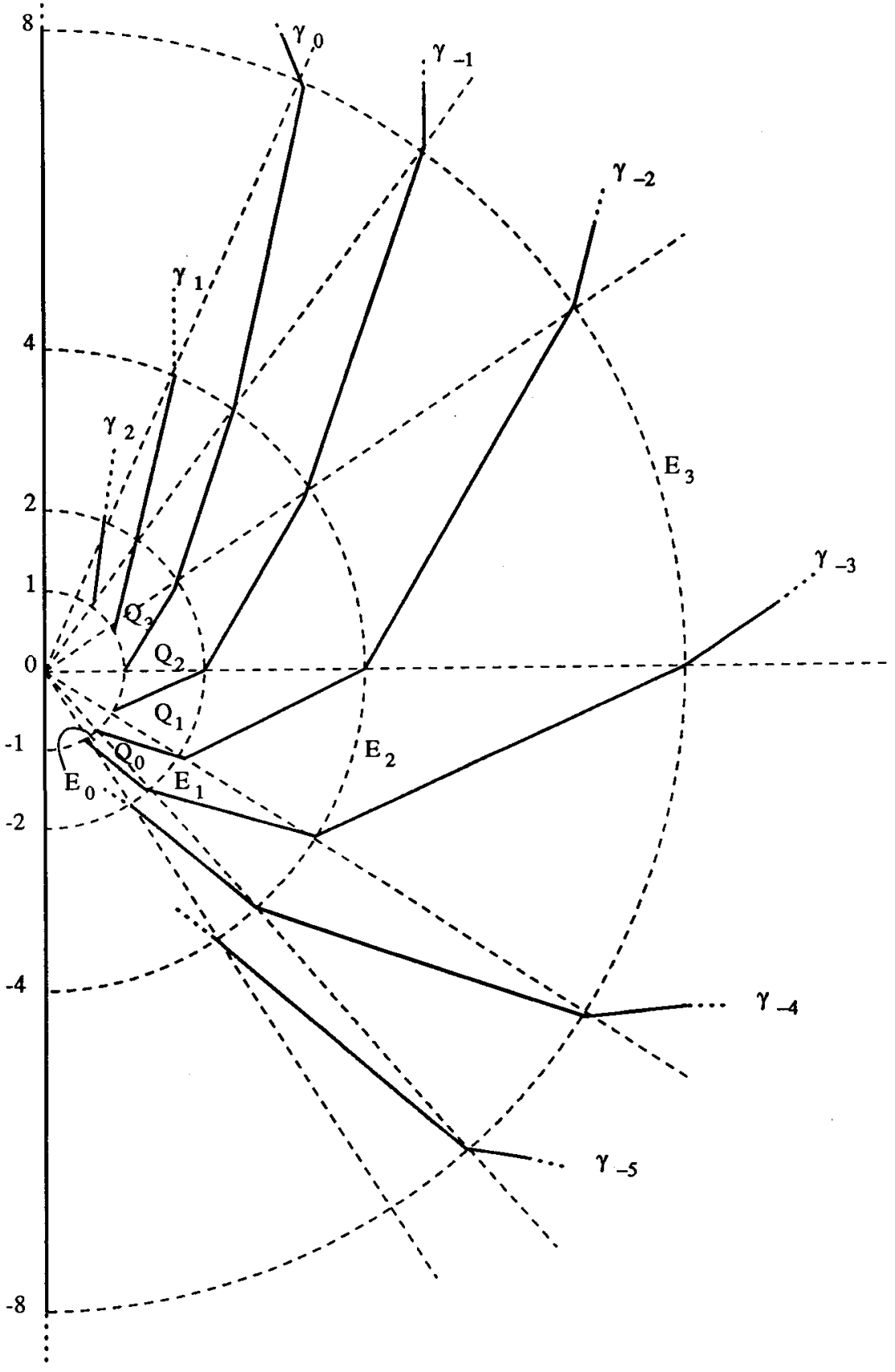

Figure 1. Spiral domain 
Note that, for $n<0$, a path going to infinity while staying between the curves $\gamma_{n-1}$ and $\gamma_{n}$ first encounters arcs of increasing angular size, until it reaches, on the circle of radius $2^{-n}$, the arc $\left(2^{-n} x_{0} ; 2^{-n} x_{-1}\right)$ of maximal angular size $\theta_{1}>0$, then arcs of angular size eventually decreasing to 0 . So $G$ does not contain a twisted sector. However, if we are given $\epsilon>0$ and a certain frequency, it is impossible to find a distance $R>0$ from the origin such that any path in $G \backslash B(0, R)$, going to infinity, encounters openings of angular size smaller than $\epsilon$, at that given frequency. Hence, this example is very different from the example with vertical slits discussed above.

Moreover, in this example $G$ is not strictly starlike and each iterate $\phi_{n}$ of the map $\phi=\sigma^{-1}(\lambda \sigma)$ has infinitely many angular derivatives at points where $\left|\phi_{n}\right|=1$, for all $n>0$. Therefore, $G$ does not satisfy any of the hypothesis required to apply [9] results (see Section 1). Nevertheless, by Theorem 1.2, we still can conclude that $\sigma \in \bigcap_{p<\infty} H^{p}$. To see why this might work, we examine this example more carefully. Recall that a way of estimating harmonic measure $(\omega)$ is to use extremal distance $(d)$, because of the inequality $\omega \leq C e^{-\pi d}$ due to Beurling. Hence the rate of growth of $d$ will give us information on the rate of decay of $\omega$. Now, we restrict our attention to a single channel going to infinity, enclosed between the curves $\gamma_{n-1}$ and $\gamma_{n}$. Then, for all $k \geq 0$, the extremal distance between the arc $E_{k}=\left(2^{k} x_{n-1+k} ; 2^{k} x_{n+k}\right)$, on the circle of radius $2^{k}$, and the arc $E_{k+1}=$ $\left(2^{k+1} x_{n+k} ; 2^{k+1} x_{n+1+k}\right)$, on the circle of radius $2^{k+1}$, is the same as the extremal distance between the arc $F_{k}=\left(1 / 2^{k}\right) E_{k}$, on the circle of radius 1 , and the arc $G_{k}=\left(1 / 2^{k}\right) E_{k+1}$, on the circle of radius 2 , by conformal invariance. So, let $Q_{k}$ be the quadrilateral enclosed by the two arcs $F_{k}$ and $G_{k}$, and the straight segments $\left[x_{n-1+k}, 2 x_{n+k}\right] \subset \gamma_{n-1+k}$ and $\left[x_{n+k}, 2 x_{n+1+k}\right] \subset \gamma_{n+k}$; see Figure 1. By the serial rule and using the euclidean metric to estimate the extremal distance, we obtain:

$$
d\left(E_{0}, E_{m}\right) \geq \sum_{k=0}^{m-1} d\left(E_{k}, E_{k+1}\right)=\sum_{k=0}^{m-1} d\left(F_{k}, G_{k}\right) \geq \sum_{k=0}^{m-1} 1 / \operatorname{area}\left(Q_{k}\right) .
$$

Then, since the quadrilaterals $Q_{k}$ are all disjoint and all fit in the annulus $\{1 \leq$ $|z| \leq 2\}$, by Cauchy-Schwarz inequality:

$$
\sum_{k=0}^{m-1} 1 / \operatorname{area}\left(Q_{k}\right) \geq m^{2} /\left(\sum_{k=0}^{m-1} \operatorname{area}\left(Q_{k}\right)\right) \geq 3 \pi m^{2} .
$$

Therefore, $d\left(E_{0}, E_{m}\right) \geq 3 \pi m^{2}$. Also, notice that this estimate doesn't depend on the channel we fixed. So, using the "conditional probability method" (see Section 3.1 ) to add the harmonic measure up, and recalling that $\alpha \sim 2^{m}$, we obtain that, for all $\epsilon>0$ :

$$
\omega(0, \partial(\alpha U), G) \leq C_{1} e^{-C_{2}(\log \alpha) / \epsilon},
$$

where $C_{1}=C_{1}(\epsilon)>0$ and $C_{2}$ is a positive absolute constant. Therefore, the harmonic measure $\omega(0, \partial(\alpha U), G)$ is certainly integrable against $\alpha^{p}=e^{p \log \alpha}$, for every $p<\infty$, which is what is needed to bound the $H^{p}$-norms of $\sigma$. In the following, we will show how this line of proof can be generalized to any region $G$ with $\lambda G \subset G$.

Finally, note that since the intersection of $G$ with circles of arbitrary radius always contains an arc of angular size $\theta_{1}>0$, methods like Carleman's or Tsuji's direct estimates of the extremal distance, using integrals of the form $\int_{1}^{R}\left(\Theta^{\star}(t)\right)^{-1} d t$, with $\Theta^{\star}(t)=\max \{|E|: \quad E$ subarc of $G \cap\{|z|=t\}\}$, where $|E|$ is the angular 
measure of $E$, don't work in this case. See [6], Theorem 3.1, for an explicit estimate of the Hardy class of $\sigma$ using Tsuji's method.

\section{Proof of Theorem 1.2}

3.1. $H^{p}$ norms and harmonic measure. Condition (c) of Theorem 1.2 implies (b), by Theorem 3.1 of [9]. Also, (b) clearly implies (a), because a k-invariant twisted sector is, in particular, a twisted sector. So, we only need to show that (a) implies (c), i.e. that if $G$ does not contain a $k$-invariant twisted sector for any $k \geq 1$, then $\sigma \in \bigcap_{p<\infty} H^{p}$.

We will bound the $H^{p}$-norms of $\sigma$ by estimating the distribution function of $\sigma$ using harmonic measure on the region $G$. Since $\sigma$ is a univalent function on $U, \sigma$ belongs to the Hardy space $H^{p}(U)$, for all $0<p<1 / 2$; see [3], Theorem 3.16, p. 50. Therefore, $\sigma$ has a radial limit at almost every point of $\partial U$. Moreover, $\sigma \in H^{p}$, $p>0$, if and only if $\|\sigma\|_{p}^{p}=\int_{0}^{2 \pi}\left|\sigma\left(e^{i \theta}\right)\right|^{p} d \theta<\infty$; see [3], Theorem 2.11, p. 28. For $\alpha \in(0, \infty)$, let $E_{\alpha}=\left\{e^{i \theta} \in \partial U:\left|\sigma\left(e^{i \theta}\right)\right|>\alpha\right\}$. Then, by Fubini's Theorem, we have:

$$
\|\sigma\|_{p}^{p}=p \int_{0}^{\infty} \alpha^{p-1}\left|E_{\alpha}\right| d \alpha=2 \pi p \int_{0}^{\infty} \alpha^{p-1} \omega\left(0, E_{\alpha}, U\right) d \alpha .
$$

Now, let $F_{\alpha}$ be the set $\{w \in U:|\sigma(w)|=\alpha\}$, and let $\Omega_{\alpha}$ be the component of $\{w \in U:|\sigma(w)|<\alpha\}$ that contains the origin. Then, $F_{\alpha}$ consists of countably many analytic Jordan arcs beginning and ending on $\partial U$. Also, $\partial \Omega_{\alpha} \cap E_{\alpha}=\emptyset$ and $\partial \Omega_{\alpha} \cap U \subset F_{\alpha}$. So, $\omega\left(0, E_{\alpha}, U\right) \leq \omega\left(0, F_{\alpha}, \Omega_{\alpha}\right)$. By conformal invariance, $\omega\left(0, F_{\alpha}, \Omega_{\alpha}\right)=\omega(0, \partial(\alpha U), G(0, \alpha))$, where $G(0, \alpha)$ is the component of $G \cap(\alpha U)$ that contains the origin. Thus,

$$
\omega\left(0, E_{\alpha}, U\right) \leq \omega(0, \partial(\alpha U), G(0, \alpha)) .
$$

At this point, we need to introduce some further notations to simplify the matter. In order to estimate $\omega(0, \partial(\alpha U), G(0, \alpha))$ we will break down $G(0, \alpha)$ into a finite sequence of subregions depending on the powers of $|\lambda|^{-1}$. Set $r=|\lambda|^{-1}>1$, and, for all $n \geq 0$, let $U_{n}=r^{n} U, T_{n}=\partial U_{n}$, and let $G_{n}=G\left(0, r^{n}\right)$ be the component of $G \cap U_{n}$ containing the origin. The sets $G_{n}$ are simply connected and increase with $n$. Therefore, if $\alpha \in(0, \infty)$ is fixed, and $n \geq 0$ is an integer such that $r^{n}<\alpha$, then $G_{n} \subset G(0, \alpha)$. By comparing the respective boundary behaviors, we obtain the "conditional probability" inequality:

$$
\omega(0, \partial(\alpha U), G(0, \alpha)) \leq \omega\left(0, T_{n}, G_{n}\right) \cdot \sup _{\zeta \in T_{n}} \omega(\zeta, \partial(\alpha U), G(0, \alpha)) .
$$

This follows from the following facts: $G_{n} \subset G(0, \alpha) ; \partial G_{n} \cap\left[G(0, \alpha) \cap T_{n}\right]$ is a union of open $\operatorname{arcs}$ of $T_{n}$ and, for $\zeta \in \partial G_{n} \cap\left[G(0, \alpha) \cap T_{n}\right], \omega\left(\zeta, T_{n}, G_{n}\right)=1$, so the inequality clearly holds in this case; $\partial G_{n} \backslash\left[G(0, \alpha) \cap T_{n}\right] \subset \partial G(0, \alpha) \cap(\alpha U)$, so that the upper limit of the left-hand side is always 0 , when tending to $\zeta \in \partial G_{n} \backslash\left[G(0, \alpha) \cap T_{n}\right]$.

Claim 3.1. Under the hypothesis of Theorem 1.2 (a), given $\epsilon>0$, there are integers $N \geq 1$ and $K \geq 1$ so that, for all $n \geq N$ :

$$
\sup _{\zeta \in T_{n}} \omega\left(\zeta, T_{n+K}, G_{n+K}\right) \leq e^{-K / \epsilon}<1 .
$$


Assume this claim for the moment. Then, given $\alpha \in\left(r^{N}, \infty\right)$, choose $H \geq 0$ such that $r^{N+H K}<\alpha<r^{N+(H+1) K}$. Iterating the conditional probability estimate described above, we obtain that:

$$
\omega(0, \partial(\alpha U), G(0, \alpha)) \leq \prod_{h=0}^{H-1} \sup _{\zeta \in T_{N+h K}} \omega\left(\zeta, T_{N+(h+1) K}, G_{N+(h+1) K}\right) \leq e^{-(H K) / \epsilon} .
$$

From our choice of $H$ we deduce that $-H K<(N+K)-(\log r)^{-1} \log \alpha$. Let $C_{1}=(\log r)^{-1}$. Then:

$$
\omega(0, \partial(\alpha U), G(0, \alpha)) \leq e^{(N+K) / \epsilon} \alpha^{-C_{1} / \epsilon} .
$$

Now, fix $p \in(0, \infty)$. Choose $\epsilon>0$ so that $p<C_{1} / \epsilon$. Then, by (3.1), (3.2), (3.3), and Claim 3.1, there are integers $N \geq 1$ and $K \geq 1$ such that:

$$
\|\sigma\|_{p}^{p} \leq 2 \pi p\left[\int_{0}^{r^{N}} \alpha^{p-1} d \alpha+e^{(N+K) / \epsilon} \int_{r^{N}}^{\infty} \alpha^{p-1-C_{1} / \epsilon} d \alpha\right] .
$$

But:

$$
\int_{r^{N}}^{\infty} \alpha^{p-1-C_{1} / \epsilon} d \alpha=\frac{1}{p-C_{1} / \epsilon}\left[\lim _{\alpha \rightarrow \infty} \alpha^{p-C_{1} / \epsilon}-r^{N\left(p-C_{1} / \epsilon\right)}\right]<\infty,
$$

because $\lim _{\alpha \rightarrow \infty} \alpha^{p-C_{1} / \epsilon}=0$, by our choice of $\epsilon$. This proves $\sigma \in H^{p}$ for all $p<\infty$. Therefore, we only need to prove Claim 3.1.

3.2. The invariant subset of $G$. Instead of proving Claim 3.1 directly on $G$, we will show that it holds on a subset of $G$ that, roughly speaking, contains the "asymptotic" behavior of $G$ at infinity. Then, by an approximation argument we'll obtain the full claim. Let $V=\operatorname{int}\left(\bigcap_{n \geq 0} \lambda^{n} G\right)$. The set $V$ is contained in $G$, and if $V$ is non-empty the connected components of $V$ are simply connected. In order to have a better understanding of the set $V$ it is worth seeing what the components of $V$ look like in the cases examined in Section 2 or in the case when $G$ contains a straight sector, and how $\lambda$ acts on them. The next lemma describe some interesting properties of the set $V$.

Lemma 3.2. The set $V$ is completely invariant, i.e. $\lambda V=V=\lambda^{-1} V$, and $0 \notin V$. Moreover, if $V_{j}$ is a connected component of $V$, then $\lambda V_{j}=V_{l}$, where $V_{l}$ is a component of $V$.

Proof. Since $\lambda G \subset G, G \cap \lambda G=\lambda G$. So:

$$
\begin{aligned}
V & =\operatorname{int}\left(\bigcap_{n \geq 0} \lambda^{n} G\right)=\operatorname{int}\left(\bigcap_{n \geq 1} \lambda^{n} G\right) \\
& =\operatorname{int}\left(\bigcap_{n \geq 0} \lambda^{n+1} G\right)=\lambda \operatorname{int}\left(\bigcap_{n \geq 0} \lambda^{n} G\right)=\lambda V .
\end{aligned}
$$

Thus $V=\lambda V$. Multiplying both sides by $\lambda^{-1}$ we obtain that $V=\lambda^{-1} V$. Now, suppose that $0 \in V$. Then, there is $\delta>0$ small enough so that $B(0, \delta) \subset V$. Hence, for all $n>0, \lambda^{-n} B(0, \delta)=B\left(0, r^{n} \delta\right) \subset V$, i.e. $V$ contains the complex plane, but this is a contradiction.

Let $V_{j}$ be a component of $V$. Then, $\lambda V_{j} \subset \lambda V=V$, and $\lambda V_{j}$ is connected. So, there is a component $V_{l}$ of $V$ such that $\lambda V_{j} \subset V_{l}$. Likewise, $\lambda^{-1} V_{l} \subset \lambda^{-1} V=V$, $\lambda^{-1} V_{l}$ is connected, and $V_{j} \subset \lambda^{-1} V_{l}$. Therefore, $V_{j}=\lambda^{-1} V_{l}$, and $\lambda V_{j}=V_{l}$. 
This lemma shows that the collection $\left\{V_{j}\right\}$ of all connected components of $V$ can be divided into orbits of the form $\left\{\lambda^{k} V_{j_{0}}\right\}_{k=-\infty}^{+\infty}$, where $V_{j_{0}}$ is some fixed component of $V$. An orbit can be either infinite, in which case $\lambda^{k} V_{j_{0}} \cap \lambda^{k^{\prime}} V_{j_{0}}=\emptyset$, for all $k \neq k^{\prime}$; or finite, in which case $V_{j_{0}}$ is invariant for some power of $\lambda$, i.e. there is an integer $k$ such that $\lambda^{k} V_{j_{0}}=V_{j_{0}}$.

Proposition 3.3. The set $V$ has a component that is invariant for some power $k$ of $\lambda$ if and only if $G$ contains a k-invariant twisted sector.

Proof. First suppose that $G$ contains a $k$-invariant twisted sector $S=S_{\epsilon}[\gamma]$, for some $\epsilon>0$ and some curve $\gamma$ from 0 to $\infty$ in $\mathbf{C} \backslash\{0\}$ such that $\lambda^{k} \gamma=\gamma$. Then, $S=S_{\epsilon}\left[\left(\lambda^{k}\right)^{n} \gamma\right]=\lambda^{n k} S \subset \lambda^{n k} G$, for all $n \geq 0$. So, since $S$ is open, $S \subset V$. Note that $S$ is connected, because $\gamma$ itself is connected and $\gamma \subset \mathbf{C} \backslash\{0\}$. Therefore there is a component $V_{j}$ of $V$ containing $S$; and $\lambda^{k} V_{j}=V_{j}$.

Conversely, suppose there is a component $V_{j}$ of $V$ such that $\lambda^{k} V_{j}=V_{j}$, for some $k$. Pick $z_{0} \in V_{j}$. By Lemma 3.2, $z_{0} \neq 0$. Also, $\lambda^{k} z_{0} \in V_{j}$. So, there is a curve $\gamma_{0} \subset$ $V_{j}$ connecting $z_{0}$ to $\lambda^{k} z_{0}$. Choose $\epsilon>0$ small enough so that $S_{\epsilon}\left[\gamma_{0}\right] \subset V_{j}$. Then, let $\gamma=\bigcup_{n=-\infty}^{+\infty}\left(\lambda^{k}\right)^{n} \gamma_{0}$. Note that $\gamma$ is a curve from 0 to $\infty$ in $\mathbf{C} \backslash\{0\}$ such that $\lambda^{k} \gamma=\gamma$. Moreover, $S_{\epsilon}[\gamma]=\bigcup_{n=-\infty}^{+\infty} S_{\epsilon}\left[\left(\lambda^{k}\right)^{n} \gamma_{0}\right]=\bigcup_{n=-\infty}^{+\infty}\left(\lambda^{k}\right)^{n} S_{\epsilon}\left[\gamma_{0}\right] \subset V_{j} \subset G$. So, $G$ contains a $k$-invariant twisted sector.

In view of the last proposition, to prove Theorem 1.2, it's enough to show that if $\lambda^{k} V_{j} \cap V_{j}=\emptyset$ for every $k \geq 1$ and every component $V_{j}$ of $V$, then Claim 3.1 holds, hence $\sigma \in \bigcap_{p<\infty} H^{p}$.

3.3. Estimate of the harmonic measure for the invariant set $V$. We will show that Claim 3.1 holds if we restrict ourselves to the set $V$.

Proposition 3.4. If $\lambda^{k} V_{j} \cap V_{j}=\emptyset$ for every $k \geq 1$ and every component $V_{j}$ of $V$, then, given $\epsilon>0$, there is an integer $K \geq 1$ such that for every component $\Omega$ of $V \cap U_{K}$, with $\Omega \cap T_{0} \neq \emptyset$, and every $\zeta \in \Omega \cap T_{0}$ :

$$
\omega\left(\zeta, T_{K}, \Omega\right) \leq e^{-(2 K) / \epsilon}<1 .
$$

Proof. First we assume that each component of $V$ is bounded by an analytic Jordan curve. Fix $k \geq 1$ and fix a component $V_{j}$ of $V$. Since $\partial V_{j}$ is an analytic Jordan curve distinct from $T_{k}, \partial V_{j} \cap T_{k}$ consists of a finite number of points. Assume that $\Omega$ is a component of $V_{j} \cap U_{k}$ such that $\Omega \cap T_{0} \neq \emptyset$, and fix $\zeta \in \Omega \cap T_{0}$. Then, $\partial \Omega \cap T_{k}$ consists of a finite union of arcs. To obtain our estimate we will use Beurling's free arc method. Since $T_{0}=\partial U$ and, by Lemma 3.2, $0 \notin \Omega$, we can find an $\operatorname{arc} \gamma$ in $U$, connecting $\zeta$ to $\partial \Omega$. Let $d=d_{\Omega \backslash \gamma}\left(\gamma, T_{k}\right)$ be the extremal distance in the set $\Omega \backslash \gamma$, between $\gamma$ and $T_{k}$. Note that $\partial \Omega$ is connected because $\Omega$ is simply connected. Then, by Theorem 3.3 in Chap. 3 of [5], or [2], p. 372:

$$
\omega\left(\zeta, T_{k}, \Omega\right) \leq \frac{8}{\pi} e^{-\pi d}
$$

We need to estimate the distance $d_{\Omega \backslash \gamma}\left(\gamma, T_{k}\right)$. Given integers $m<n$, let $\Omega_{m, n}=$ $\Omega \cap\left(U_{n} \backslash c l\left(U_{m}\right)\right)$. By the serial rule, see [5], Chap. 3:

$$
d_{\Omega \backslash \gamma}\left(\gamma, T_{k}\right) \geq \sum_{n=0}^{k-1} d_{\Omega_{n, n+1}}\left(T_{n}, T_{n+1}\right) .
$$


We estimate each term of the sum using the euclidean metric:

$$
d_{\Omega_{n, n+1}}\left(T_{n}, T_{n+1}\right) \geq \frac{\left(r^{(n+1)}-r^{n}\right)^{2}}{\operatorname{Area}\left(\Omega_{n, n+1}\right)}=\frac{(r-1)^{2}}{|\lambda|^{2 n} \operatorname{Area}\left(\Omega_{n, n+1}\right)}
$$

Let $C=(r-1)^{2}$. Note that $|\lambda|^{2 n} \operatorname{Area}\left(\Omega_{n, n+1}\right)=\operatorname{Area}\left(\lambda^{n} \Omega_{n, n+1}\right)$. Now, $\Omega$ is contained in a component of $V$. So, by our hypothesis, $\lambda^{n} \Omega_{n, n+1} \cap \lambda^{m} \Omega_{m, m+1}=\emptyset$, for all $n \neq m$, and $\lambda^{n} \Omega_{n, n+1} \subset U_{1} \backslash U$, for all $n \geq 0$. Hence, $\sum_{n=0}^{k-1} \operatorname{Area}\left(\lambda^{n} \Omega_{n, n+1}\right) \leq$ Area $\left(U_{1} \backslash U\right)=\pi\left(r^{2}-1\right)=M<\infty$. Then, by the Cauchy-Schwarz inequality:

$$
\sum_{n=0}^{k-1} \frac{1}{\operatorname{Area}\left(\lambda^{n} \Omega_{n, n+1}\right)} \geq \frac{k^{2}}{M}
$$

Thus, $d_{\Omega \backslash \gamma}\left(\gamma, T_{k}\right) \geq(C / M) k^{2}$, and $\omega\left(\zeta, T_{k}, \Omega\right) \leq \frac{8}{\pi} e^{-\pi(C / M) k^{2}}$. Now, given $\epsilon>0$, we want $\frac{8}{\pi} e^{-\pi(C / M) k^{2}} \leq e^{-(3 k) / \epsilon}$. This inequality holds for $k$ sufficiently large, so there is $K$ such that for every component $\Omega$ of $V \cap U_{K}$ with $\Omega \cap T_{0} \neq \emptyset$ and every $\zeta \in \Omega \cap T_{0}, \omega\left(\zeta, T_{K}, \Omega\right) \leq e^{-(3 K) / \epsilon}<1$.

Here, we assumed that $\partial V_{j}$ is analytic. But note that each component $V_{j}$ of $V$ is simply connected. So, we can exhaust it with an increasing sequence of regions $V_{j}^{(n)}$ bounded by an analytic Jordan curve. Then, the harmonic functions $\omega\left(\cdot, T_{K}, V_{j}^{(n)}\right)$ converge uniformly on compact subsets of $V_{j}$ to $\omega\left(\cdot, T_{K}, V_{j}\right)$. Therefore, applying Beurling's projection theorem, see [1], Thm. 3.6, p. 43, to points $\zeta \in T_{0}$ that are close to $\partial V_{j}$, we conclude that $\omega\left(\zeta, T_{K}, \Omega\right) \leq e^{-(2 K) / \epsilon}<1$, for every component $\Omega$ of $V \cap U_{K}$ such that $\Omega \cap T_{0} \neq \emptyset$, and every $\zeta \in \Omega \cap T_{0}$.

Now we want to deduce the same estimate on the set $G$ using the fact that $G$ looks like $V$ at infinity.

3.4. Estimate on $G$ via approximation. According to Claim 3.1 we need to estimate $\omega\left(\zeta, T_{n+K}, G_{n+K}\right)$, for all $\zeta \in T_{n}$, for some given $K \geq 1$, and for all $n \geq 0$. By conformal invariance, we can just look at $\omega\left(\zeta, T_{K}, \lambda^{n} G_{n+K}\right)$, for all $\zeta \in T_{0}$. In the following we will always assume $\epsilon>0$ to be fixed, and $K$ to be given as in Proposition 3.4. Note that $\lambda^{n} G_{n+K}$ decreases with $n$. In fact, $\lambda^{n} G_{n+K}$ is the component of $\lambda^{n} G \cap U_{K}$ that contains the origin. So, since $\lambda^{n+1} G \cap U_{K}$ is contained in $\lambda^{n} G \cap U_{K}$, we must have $\lambda^{n+1} G_{n+1+K} \subset \lambda^{n} G_{n+K}$. In view of this fact, we set $W_{K}=\operatorname{int}\left(\bigcap_{n \geq 0} \lambda^{n} G_{n+K}\right)$.

Lemma 3.5. The set $W_{K}$ consists of components of $V \cap U_{K}$.

Proof. First notice that $\bigcap_{n \geq 0} \lambda^{n} G_{n+K} \subset \bigcap_{n \geq 0}\left(\lambda^{n} G \cap U_{K}\right)=\left(\bigcap_{n \geq 0} \lambda^{n} G\right) \cap U_{K}$. So $W_{K} \subset V \cap U_{K}$. Thus, if $W_{K}^{j}$ is a component of $W_{K}$, then there is a component $V_{j}$ of $V$, and a component $\Omega$ of $V_{j} \cap U_{K}$, such that $W_{K}^{j}$ is contained in $\Omega$.

Now, $\Omega$ is connected and contained in $\lambda^{n} G \cap U_{K}$ for all $n \geq 0$. Since $W_{K}^{j} \subset \Omega$, we have $\Omega \cap \lambda^{n} G_{n+K} \neq \emptyset$ for all $n \geq 0$. Since $G_{n+K}$ is a component of $G \cap U_{n+K}$, we must have $\Omega \subset \lambda^{n} G_{n+K}$ for all $n \geq 0$. Therefore, $\Omega \subset W_{K}$, and $\Omega=W_{K}^{j}$.

Under the assumptions of Proposition 3.4 and by Lemma 3.5, we have that $\omega\left(\zeta, T_{K}, \Omega\right) \leq e^{-(2 K) / \epsilon}<1$, for every component $\Omega$ of $W_{K}$ such that $\Omega \cap T_{0} \neq \emptyset$, and every $\zeta \in \Omega \cap T_{0}$. We want to obtain a similar statement for each $\lambda^{n} G_{n+K}$.

For all $n \geq 0$, let $u_{n}=\omega\left(\cdot, T_{K}, \lambda^{n} G_{n+K}\right)$. Since $\lambda^{n} G_{n+K}$ decrease with $n$, $u_{n}$ is a decreasing sequence of harmonic functions defined on $W_{K}$. By Harnack's 
Principle, there is a harmonic function $u$ defined on $W_{K}$ such that $u_{n}$ converges to $u$ uniformly on compact subsets of $W_{K}$. Moreover, $0 \leq u \leq 1$. Given a component $\Omega$ of $W_{K}$, we want to compare $u$ with $\omega\left(\cdot, T_{K}, \Omega\right)$.

Lemma 3.6. For $\zeta \in \partial \Omega \cap U_{K}$, $\limsup _{z \in \Omega \rightarrow \zeta} u(z)=0$. Therefore, for all $z \in \Omega$, $u(z) \leq \omega\left(z, T_{K}, \Omega\right)$.

Proof. Fix $\beta>0$. Let $\Delta$ be a small disk centered at $\zeta \in \partial \Omega \cap U_{K}$. Then, since $\Delta \not \subset W_{K}$, there is $N$ large enough so that $\left(\lambda^{N} G_{N+K}\right)^{c} \cap \Delta \neq \emptyset$; hence $\partial\left(\lambda^{N} G_{N+K}\right) \cap$ $\Delta \neq \emptyset$, because $\lambda^{N} G_{N+K} \cap \Delta \neq \emptyset$. By Beurling's projection theorem, if $\Delta$ is chosen small enough. $u_{N}(z) \leq \beta$ for all $z \in \Delta \cap \Omega$. Therefore, $u(z) \leq u_{N}(z) \leq \beta$ for all $z \in \Delta \cap \Omega$, i.e. $\lim \sup u(z)=0$ as $z \rightarrow \zeta$.

Since $0 \leq u \leq 1, u$ is a candidate for the Perron solution to the Dirichlet problem on $\Omega$ with data the characteristic function of $\partial \Omega \cap T_{K}$. Hence, $u(z) \leq \omega\left(z, T_{K}, \Omega\right)$ for all $z \in \Omega$.

By Proposition 3.4 and by Lemma 3.6, $u(z) \leq e^{-(2 K) / \epsilon}<1$, for all $z \in W_{K} \cap T_{0}$. Since $u_{n}$ converges to $u$, we claim that there is $N$ large enough so that $u_{N}(z) \leq$ $e^{-K / \epsilon}<1$, for all $z \in \lambda^{N} G_{N+K} \cap T_{0}$. Given a set $A$ and $\delta>0$, define $I_{\delta}[A]=\{z \in$ $A: d(z, \partial A) \geq \delta\}$. By Beurling's projection theorem, it follows that, if $\delta$ is chosen small enough, whenever $z \in \lambda^{n} G_{n+K} \cap T_{0}$ for $n \geq 0$, but $z \notin I_{\delta}\left[\lambda^{n} G_{n+K}\right]$, we have $u_{n}(z)=\omega\left(z, T_{K}, \lambda^{n} G_{n+K}\right) \leq e^{-K / \epsilon}$.

Lemma 3.7. Given $\delta>0$, there is $N_{0}$ large enough so that for all $n \geq N_{0}$ :

$$
I_{\delta}\left[\lambda^{n} G_{n+K}\right] \cap T_{0} \subset I_{\delta / 2}\left[W_{K}\right] \cap T_{0} .
$$

Proof. If not, for all $n \geq 0$, we can choose $m_{n}>n$ and a point $z_{m_{n}}$ contained in $I_{\delta}\left[\lambda^{m_{n}} G_{m_{n}+K}\right] \cap T_{0}$ such that $z_{m_{n}} \notin I_{\delta / 2}\left[W_{K}\right] \cap T_{0}$. Also, since $T_{0}$ is compact we can assume that $z_{m_{n}}$ converges to some $z_{0} \in T_{0}$. Then, for all $n \geq$ $0, B\left(z_{m_{n}}, \delta\right) \subset \lambda^{m_{n}} G_{m_{n}+K}$. Moreover, we can find $n_{0}$ such that for $n \geq n_{0}$, $B\left(z_{0}, 3 \delta / 4\right) \subset B\left(z_{m_{n}}, \delta\right) \subset \lambda^{m_{n}} G_{m_{n}+K}$. Since $\lambda^{n} G_{n+K}$ is a decreasing sequence of sets, $B\left(z_{0}, 3 \delta / 4\right) \subset \bigcap_{n \geq 0} \lambda^{n} G_{n+K}$, i.e. $B\left(z_{0}, 3 \delta / 4\right) \subset W_{K}$; so $z_{0} \in I_{3 \delta / 4}\left[W_{K}\right] \cap T_{0} \subset$ $\operatorname{int}\left(I_{\delta / 2}\left[W_{K}\right]\right) \cap T_{0}$. Since $z_{m_{n}}$ converges to $z_{0}$, eventually $z_{m_{n}} \in \operatorname{int}\left(I_{\delta / 2}\left[W_{K}\right]\right) \cap T_{0}$, which is a contradiction.

Now observe that $I_{\delta / 2}\left[W_{K}\right] \cap T_{0}$ is a compact subset of $W_{K}$, where $u(z)$ is uniformly bounded by $e^{-(2 K) / \epsilon}$. Since $u_{n}$ converges to $u$ uniformly on compact subsets of $W_{K}$, we can find $N>N_{0}$, such that for all $z \in I_{\delta / 2}\left[W_{K}\right] \cap T_{0}$, and all $n \geq N, u_{n}(z) \leq e^{-K / \epsilon}<1$. Then, by our choice of $\delta$ and by Lemma 3.7, $u_{n}(z) \leq e^{-K / \epsilon}<1$, for all $z \in \lambda^{n} G_{n+K} \cap T_{0}$, and all $n \geq N$. Finally, by conformal invariance, we obtain that:

$$
\omega\left(z, T_{n+K}, G_{n+K}\right) \leq e^{-K / \epsilon}<1,
$$

for all $z \in G_{n+K} \cap T_{n}$ and all $n \geq N$. Hence, we have proved Claim 3.1 and therefore Theorem 1.2.

\section{Connection With COMposition operators}

We saw in the Introduction that the map $\sigma$ can be interpreted as an eigenfunction of the composition operator $C_{\phi}$ with symbol $\phi=\sigma^{-1}(\lambda \sigma)$. The Eigenfunction Theorem, as formulated in [11], p. 94, says that if some power of the operator $C_{\phi}$ acting on $H^{2}(U)$ is compact, then $\sigma \in \bigcap_{p<\infty} H^{p}$. This theorem is proved in [11] 
using operator theory. Theorem 1.2 will provide us with a strictly geometric proof. In fact, note that if $\left(C_{\phi}\right)^{n_{0}}$ is compact on $H^{2}(U)$, then $\left(C_{\phi}\right)^{n}=C_{\phi_{n}}$ is compact for all $n \geq n_{0}$. Therefore, by a well-known necessity criterion for compactness (see [10], Theorem 2.1), $\phi_{n}$ has no angular derivatives at points where $\left|\phi_{n}\right|=1$, for all $n \geq n_{0}$. This result also uses operator theory, but in the Appendix we will produce a more straightforward proof of it. Then, by the next proposition, it follows easily that $G$ cannot contain a twisted sector, and by Theorem 1.2, $\sigma \in \bigcap_{p<\infty} H^{p}$. Hence, we have proved the Eigenfunction Theorem.

Proposition 4.1. The set $G$ contains a twisted sector if and only if there exists $K \geq 1$ such that $\phi_{K}$ has an angular derivative at a boundary fixed point.

Proof. One direction is proved in Proposition 3.3 of [9], namely, if $\phi_{K}$ has an angular derivative at a boundary fixed point, then $G$ contains a twisted sector. Conversely, suppose that $G$ contains a twisted sector. Then, by Theorem 1.2, $G$ contains a $K$-invariant twisted sector for some $K \geq 1$. Note that this can also be proved directly by showing that if $G$ contains a twisted sector, then it contains countably many twisted sectors, because $\lambda G \subset G$, and therefore $V$ must have a non-empty invariant component for some power $K$ of $\lambda$, which, by Proposition 3.3, gives rise to a $K$-invariant twisted sector. So, let $\gamma$ be a curve from 0 to $\infty$ in $\mathbf{C} \backslash\{0\}$, such that $\lambda^{K} \gamma=\gamma$ and $S_{\epsilon}[\gamma] \subset G$. Pick $z_{0} \in \gamma, z_{0} \neq 0$. Then, $z_{n}=\lambda^{-n K} z_{0} \in \gamma$ is a sequence converging to infinity as $n$ goes to infinity. So, if we set $w_{n}=\sigma^{-1}\left(z_{n}\right)$, for all $n \geq 0, w_{n}$ converges to $\partial U$. Let $\rho$ be the hyperbolic metric, and $\gamma_{0}$ a piece of the curve $\gamma$ that connects $z_{0}$ to $z_{1}$. Then:

$$
\begin{aligned}
\rho_{U}\left(w_{n}, w_{n+1}\right) & =\rho_{G}\left(z_{n}, z_{n+1}\right) \leq 2 \int_{\lambda^{-n K} \gamma_{0}} \frac{|d z|}{\delta_{G}(z)} \\
& \leq 2 \int_{\lambda^{-n K} \gamma_{0}} \frac{|d z|}{\epsilon|z|}=\frac{2}{\epsilon} \int_{0}^{1} \frac{|\lambda|^{-n K}\left|\gamma_{0}^{\prime}(t)\right| d t}{|\lambda|^{-n K}\left|\gamma_{0}(t)\right|} \\
& =\frac{2}{\epsilon} \int_{\gamma_{0}} \frac{|d z|}{|z|}=C_{0} .
\end{aligned}
$$

Therefore:

$$
\left|\frac{w_{n+1}-w_{n}}{1-\overline{w_{n+1}} w_{n}}\right| \leq \frac{e^{C_{0}}-1}{e^{C_{0}}+1}=C_{1}<1
$$

So:

$$
\begin{aligned}
0<1-C_{1}^{2} & \leq 1-\left|\frac{w_{n+1}-w_{n}}{1-\overline{w_{n+1}} w_{n}}\right|^{2}=\frac{\left(1-\left|w_{n}\right|^{2}\right)\left(1-\left|w_{n+1}\right|\right)^{2}}{\left|1-\overline{w_{n+1}} w_{n}\right|^{2}} \\
& \leq 4 \frac{\left(1-\left|w_{n}\right|\right)\left(1-\left|w_{n+1}\right|\right)}{\left(1-\left|w_{n}\right|\right)^{2}}=4 \frac{1-\left|w_{n+1}\right|}{1-\left|w_{n}\right|} .
\end{aligned}
$$

Now, note that $w_{n}=\sigma^{-1}\left(z_{n}\right)=\sigma^{-1}\left(\lambda^{K} z_{n+1}\right)=\phi_{K}\left(\sigma^{-1}\left(z_{n+1}\right)\right)=\phi_{K}\left(w_{n+1}\right)$. Then $\left(1-\left|\phi_{K}\left(w_{n+1}\right)\right|\right) /\left(1-\left|w_{n+1}\right|\right) \leq 4 /\left(1-C_{1}^{2}\right)<\infty$, for all $n \geq 0$. By Lemma 4.2 (b) of [9], $w_{n}$ converges to a point $\zeta \in \partial U$, and since $w_{n}=\phi_{K}\left(w_{n+1}\right)$, by Julia's Theorem, (see [11], p. 63), $\zeta$ is a boundary fixed point of $\phi_{K}$ and by the Julia-Carathéodory Theorem (see [11], p. 57), $\phi_{K}$ has an angular derivative at $\zeta$. 


\section{The NO TWISTED SECTOR CONDITION}

We have seen that under the assumption that the univalent map $\sigma: U \longrightarrow G$ satisfies $\lambda G \subset G$ for some $0<|\lambda|<1$, i.e. $\sigma$ is an eigenfunction of the composition operator $C_{\phi}$, with symbol $\phi=\sigma^{-1}(\lambda \sigma)$, the properties (a), (b), and (c) of Theorem 1.2 are equivalent and can therefore be referred to as a unique condition, abbreviated $(N T S)$. In the following we want to describe some further characterizations of this condition.

Let us form the Riemann surface $\mathcal{S}$ obtained from $\mathbf{C} \backslash\{0\}$ by identifying $z_{0}$ and $z_{1}$ if $z_{0}=\lambda z_{1}$. Note that $\mathcal{S}$ is homeomorphic to a torus and can be represented by the annulus $A=\operatorname{cl}\left(U_{1}\right) \backslash U_{0}$, where we identify $z_{0} \in T_{0}$ and $z_{1} \in T_{1}$ if $z_{0}=\lambda z_{1}$, and write $z_{0} \sim z_{1}$. Let $p: \mathbf{C} \backslash\{0\} \longrightarrow \mathcal{S}$ be the canonical projection. Consider the set $V$ that we defined in Section 3.2. By Lemma 3.2, $V$ is saturated, i.e. $p^{-1}(p(V))=V$. So $\tilde{V}=p(V)$ is an open subset of $\mathcal{S}$, and its connected components are in oneto-one correspondence with the orbits of components of $V$ (see Section 3.2). We claim that the $(N T S)$ condition is equivalent to the fact that each component of $\tilde{V}$ is simply connected. Note that $\tilde{V}$ is $V \cap\left(A \backslash T_{0}\right)$ with the appropriate topology. Pick $z_{0} \in V \cap\left(A \backslash T_{0}\right)$. We can assume that $z_{0} \in\left[1, \lambda^{-1}\right]$. Let $\gamma_{1}(\theta)=e^{i \theta} z_{0}$, for $\theta \in[0,2 \pi]$, and $\gamma_{2}$ be the loop starting at $z_{0}$ that goes through $\lambda^{-1} \sim 1$ once, along the segment $\left[1, \lambda^{-1}\right]$. These two loops generate the fundamental group of $\mathcal{S}$. First notice that no loops based at $z_{0}$ and contained in $\tilde{V}$ can be homotopic to $\gamma_{1}$. In fact, every such loop $\gamma$ must wind once around the origin. Thus, $\lambda^{-n} \gamma$ is a countable family of curves contained in $G$ that winds once around the origin and such that $U_{n-1} \cap \lambda^{-n} \gamma=\emptyset$. Since $G$ is simply connected, $U_{n} \subset G$ for all $n \geq 0$, i.e. $G=\mathbf{C}$, which is a contradiction. In this setting, we can show that the set $\tilde{V} \subset \mathcal{S}$ contains a non-trivial loop based at $z_{0}$ homotopic in $\mathcal{S}$ to $k \gamma_{1}+m \gamma_{0}$, for some $k \geq 1$ and some integer $m$, if and only if $G$ contains a $k$-invariant twisted sector. The proof of this fact is too lengthy, considering the purposes of this paper.

On the other hand, we can strengthen the requirement that $G$ contains no twisted sector by saying that $G$ contains arbitrary long tubes if there is $\epsilon>0$ such that for all $L>0$, there is $R>0$, and a curve $\gamma$ connecting $R \partial U$ to $(R+L) \partial U$, such that $S_{\epsilon}[\gamma] \subset G$. Then, we claim that $G$ contains arbitrary long tubes if and only if $G$ contains a $k$-invariant twisted sector for some $k \geq 1$. Again, the proof of this is postponed to a future paper.

As a summary, we have seen that a univalent map on $U$, such that $\lambda G \subset G$ for some $\lambda, G=\sigma(U)$, satisfies the $(N T S)$ condition if and only if one of the following holds:

1. The set $G$ does not contain a $k$-invariant twisted sector for any $k \geq 1$.

2. The set $G$ does not contain a twisted sector.

3. The set $G$ does not contain tubes of arbitrary long length.

4. The set $V=\operatorname{int}\left(\bigcap_{n \geq 0} \lambda^{n} G\right)$ does not have invariant components for any power $k \geq 1$ of $\lambda$.

5. The set $\tilde{\tilde{V}} \subset \mathcal{S}$ is contractible to a discrete set of points.

6. The map $\phi_{k}=\sigma^{-1}\left(\lambda^{k} \sigma\right)$ does not have an angular derivative at a boundary fixed point for any $k \geq 1$.

7. $\sigma \in \bigcap_{p<\infty} H^{p}$.

8. For $1 \leq p<\infty, \sigma$ is an eigenfunction of the bounded linear operator $C_{\phi}$ acting on $H^{p}(U)$. 
We saw that compactness of some power of the composition operator $C_{\phi}$ implies that its eigenfunction $\sigma$ satisfies the $(N T S)$ condition.

Open Question. What operator theoretic property of $C_{\phi}$, which is implied by compactness, is equivalent to the fact that $\sigma$ has the (NTS) property?

Added in Proof. This question has been recently answered in [7]: $\sigma$ satisfies the $(N T S)$ condition if and only if $C_{\phi}$ is a Riesz operator.

\section{Appendix: A Different proof for the Lower estimate OF THE ESSENTIAL NORM OF A COMPOSITION OPERATOR}

Let $U$ be the unit disc and $\phi$ be an analytic map of $U$ into itself. The map $\phi$ induces an operator on the Hilbert space $H^{2}(U)$ by precomposition: $C_{\phi}(f)=f \circ \phi$. The essential norm of $C_{\phi}$ is its distance, in the operator norm, from the closed subspace of compact operators acting on $H^{2}(U)$. Also, the Nevanlinna counting function on $U$ is defined as follows: $N_{\phi}(w)=0$ if $w \notin \phi(U)$, and $N_{\phi}(w)=\sum \log \left(1 /\left|z_{j}\right|\right)$ if $\left\{z_{j}\right\}=\phi^{-1}(w)$. In [12] it is shown that if $\liminf _{|w| \rightarrow 1}(\log (1 /|w|)) / N_{\phi}(w)=c<$ $\infty$, then the essential norm of the operator $C_{\phi}$, denoted by $\left\|C_{\phi}\right\|_{e}$, is equal to $1 / \sqrt{c}$. The fact that $1 / \sqrt{c}$ is an upper bound for $\left\|C_{\phi}\right\|_{e}$ is an easy computation, while, to show that it is also a lower bound, [12] introduces some further machinery, i.e. studies the averaging property of the Nevanlinna counting function. We will show that $1 / \sqrt{c}$ is a lower bound for $\left\|C_{\phi}\right\|_{e}$ in a different and more direct way.

Theorem 6.1. Suppose that:

$$
\liminf _{|w| \rightarrow 1} \frac{\log \frac{1}{|w|}}{N_{\phi}(w)}=c<\infty .
$$

Then, $\left\|C_{\phi}\right\|_{e} \geq 1 / \sqrt{c}$.

Proof. Fix $\epsilon>0$. Choose $\left\{p_{n}\right\} \subset U$ such that: $\left(\log \left(1 /\left|p_{n}\right|\right)\right) / N_{\phi}\left(p_{n}\right) \leq c+\epsilon$, for all $n$. (This forces $p_{n} \in \phi(U)$.) We can assume, by taking a subsequence, that for all $n$ :

$$
\prod_{k \neq n}\left|\frac{p_{n}-p_{k}}{1-\overline{p_{n}} p_{k}}\right| \geq 1-\epsilon
$$

Now consider the Blaschke product

$$
B(z)=\prod_{k=0}^{\infty} \frac{\left|p_{k}\right|}{p_{k}} \frac{p_{k}-z}{1-\overline{p_{k}} z} .
$$

Without loss of generality, $p_{0}=\phi(0)$. Moreover, for all $n \geq 0$, define $B_{n}(z)=$ $\left(1-\left|p_{n}\right|^{2}\right)^{1 / 2} B(z) /\left(z-p_{n}\right)$. Then, since $|B|=1$ a.e. on $\partial U$ :

$$
\left\|B_{n}\right\|^{2}=\int_{0}^{2 \pi} \frac{1-\left|p_{n}\right|^{2}}{\left|e^{i \theta}-p_{n}\right|^{2}} \frac{d \theta}{2 \pi}=1 \quad \forall n \geq 0 .
$$

We will first show that $\left\|B_{n} \circ \phi\right\|^{2} \geq 1 / c+O(\epsilon)$, for all $n \geq 1$.

Let $\psi(z)=B_{n} \circ \phi-B_{n}\left(p_{n}\right)$; then:

$$
\|\psi\|^{2}=\left\|B_{n} \circ \phi\right\|^{2}+\left|B_{n}\left(p_{n}\right)\right|^{2}-2 \operatorname{Re} \overline{B_{n}\left(p_{n}\right)}\left(B_{n} \circ \phi(0)\right)=\left\|B_{n} \circ \phi\right\|^{2}+\left|B_{n}\left(p_{n}\right)\right|^{2},
$$


for all $n \geq 1$, because $B_{n} \circ \phi(0)=0$. Now, let $\left\{z_{j}\right\}=\phi^{-1}\left(p_{n}\right)$; we have $\psi\left(z_{j}\right)=$ $B_{n}\left(p_{n}\right)-B_{n}\left(p_{n}\right)=0$ for all $j$. Therefore:

$$
\psi(z)=\left(\prod_{j} \frac{\left|z_{j}\right|}{z_{j}} \frac{z_{j}-z}{1-\overline{z_{j}} z}\right) h(z),
$$

where $\|h\|=\|\psi\|$. Hence, $|\psi(0)|=\left|B_{n}\left(p_{n}\right)\right|=\left(\prod_{j}\left|z_{j}\right|\right)|h(0)| \leq\left(\prod_{j}\left|z_{j}\right|\right)\|h\|$, where this last inequality follows from the mean value property and the Cauchy-Schwarz inequality. Then,

$$
\|\psi\| \geq\left|B_{n}\left(p_{n}\right)\right|\left(\prod_{j} \frac{1}{\left|z_{j}\right|}\right)=\left|B_{n}\left(p_{n}\right)\right| e^{N_{\phi}\left(p_{n}\right)},
$$

so that:

$$
\begin{aligned}
\left\|B_{n} \circ \phi\right\|^{2} & \geq\left|B_{n}\left(p_{n}\right)\right|^{2}\left(e^{2 N_{\phi}\left(p_{n}\right)}-1\right) \\
& \geq\left(\prod_{k \neq n}\left|\frac{p_{n}-p_{k}}{1-\overline{p_{n}} p_{k}}\right|\right)^{2} \frac{1-\left|p_{n}\right|^{2}}{\left(1-\left|p_{n}\right|^{2}\right)^{2}} 2 N_{\phi}\left(p_{n}\right) \quad\left(e^{x}-1 \geq x\right) \\
& \geq(1-\epsilon)^{2} \frac{2}{1-\left|p_{n}\right|^{2}} \frac{1}{c+\epsilon} \log \frac{1}{\left|p_{n}\right|} \quad \text { (hypothesis and (6.1)) } \\
& \geq \frac{(1-\epsilon)^{2}}{c+\epsilon}=\frac{1}{c}+O(\epsilon) \quad\left(\log \frac{1}{x} \geq \frac{1-x^{2}}{2}\right) .
\end{aligned}
$$

Finally, we observe that $\left\{B_{n}\right\}$ and $\left\{B_{n} \circ \phi\right\}$ both converge weakly to zero in $H^{2}$. In fact, $\left\{B_{n}\right\}$ is bounded in norm, and converges to zero uniformly on compact subsets of $U$, because, given $0<r<1$ and for all $w \in r \bar{U}$ :

$$
\left|B_{n}(w)\right|<\frac{\left(1-\left|p_{n}\right|^{2}\right)^{1 / 2}}{\left|1-\overline{p_{n}} w\right|} \leq \frac{\left(1-\left|p_{n}\right|^{2}\right)^{1 / 2}}{1-r\left|p_{n}\right|} \longrightarrow 0,
$$

as $n \rightarrow \infty$. Moreover, $\left\{B_{n} \circ \phi\right\}$ is also bounded in norm, since $C_{\phi}$ is a bounded operator, and given a compact subset $E$ of $U ; \phi(E)$ is also a compact subset of $U$, so $\left\{B_{n} \circ \phi\right\}$ also converges to zero uniformly on compact subsets of $U$.

Now, fix a compact operator $K$ on $H^{2}$. Then a subsequence $\left\{K\left(B_{n_{l}}\right)\right\}$ is Cauchy, i.e. there is $l_{0}$ such that: $\left\|K\left(B_{n_{l}}\right)-K\left(B_{n_{h}}\right)\right\| \leq \epsilon$ for all $l, h \geq l_{0}$. Fix $l=l_{0}$. By weak convergence there is $h_{0}>l_{0}$ such that $\left|\left\langle B_{n_{l_{0}}}, B_{n_{h_{0}}}\right\rangle\right|<\epsilon$ and $\mid\left\langle B_{n_{l_{0}}} \circ\right.$ $\left.\phi, B_{n_{h_{0}}} \circ \phi\right\rangle \mid<\epsilon$. Set $N=n_{l_{0}}$ and $M=n_{h_{0}}$; we have:

$$
\begin{aligned}
\left\|C_{\phi}\left(B_{N}\right)-C_{\phi}\left(B_{M}\right)\right\|^{2} & \geq\left\|B_{N} \circ \phi\right\|^{2}+\left\|B_{M} \circ \phi\right\|^{2}-2\left|\left\langle B_{N} \circ \phi, B_{M} \circ \phi\right\rangle\right| \\
& \geq \frac{2}{c}+O(\epsilon)-2 \epsilon=\frac{2}{c}+O(\epsilon)
\end{aligned}
$$

and:

$$
\left.\left\|B_{N}-B_{M}\right\|^{2} \leq\left\|B_{N}\right\|^{2}+\left\|B_{M}\right\|^{2}+2\left|\left\langle B_{N}, B_{M}\right\rangle\right| \leq 2+O(\epsilon) \quad \text { (by }(6.2)\right) .
$$


So:

$$
\begin{aligned}
\left\|C_{\phi}-K\right\| & \geq \frac{\left\|\left(C_{\phi}-K\right)\left(B_{N}-B_{M}\right)\right\|}{\left\|B_{N}-B_{M}\right\|} \\
& \geq \frac{\left\|C_{\phi}\left(B_{N}\right)-C_{\phi}\left(B_{M}\right)\right\|-\left\|K\left(B_{N}\right)-K\left(B_{M}\right)\right\|}{\left\|B_{N}-B_{M}\right\|} \\
& \geq \frac{\sqrt{2 / c+O(\epsilon)}-\epsilon}{\sqrt{2+O(\epsilon)}}=\frac{1}{\sqrt{c}}+O(\epsilon) .
\end{aligned}
$$

Now, let $\epsilon$ tend to zero: $\left\|C_{\phi}-K\right\| \geq 1 / \sqrt{c}$. Also let $K$ vary among all compact operators: $\left\|C_{\phi}\right\|_{e} \geq 1 / \sqrt{c}$.

In the case when $\phi$ is a univalent map, $N_{\phi}(w)=\log (1 /|z|)$, where $w=\phi(z)$. So, the condition of Theorem 6.1 becomes

$$
\liminf _{|z| \rightarrow 1} \frac{\log (1 /|\phi(z)|)}{\log (1 /|z|)}=\liminf _{|z| \rightarrow 1} \frac{1-|\phi(z)|}{1-|z|}=c<\infty
$$

which in turn is equivalent to $\phi$ having an angular derivative at a point where $|\phi|=1$, by the Julia-Carathéodory Theorem (see [11], p.57). Thus, when $\phi$ is univalent, Theorem 6.1 says that if $\phi$ has an angular derivative at a point where $|\phi|=1$, then $\left\|C_{\phi}\right\|_{e} \neq 0$, i.e. $C_{\phi}$ is not compact.

\section{REFERENCES}

1. L. Ahlfors, Conformal Invariants, McGraw-Hill, 1973. MR 50:10211

2. L. Carleson, et al. (eds.), The collected works of Arne Beurling, Vol. 1, Birkhäuser, 1989. MR 92k:01046

3. P. Duren, Theory of $H^{p}$ spaces, Academic Press, 1970.

4. G. Königs, Recherches sur les intégrales de certaines équations functionnelles, Annales Ecole Normale Supérieure (3) 1 (1884), Supplément, 3-41.

5. J. Garnett, and D. Marshall, Harmonic measure, to appear.

6. L. Hansen, Hardy classes and ranges of functions, Michigan Math. J. 17 (1970), 235-248. MR 41:7118

7. P. Poggi-Corradini, The Hardy class of geometric models and the essential spectral radius of composition operators, preprint.

8. C. Pommerenke, Schlichte Funktionen und analytische Funktionen von beschränkter mittler Ozillation, Comm. Math. Helv. 52 (1977), 591-602. MR 56:11268

9. J. H. Shapiro, W. Smith, and D. Stegenga, Geometric models and compactness of composition operators, J. Funct. Anal. 127 (1995), 21-62. CMP 95:05

10. J. H. Shapiro and P. D. Taylor, Compact, nuclear, and Hilbert-Schmidt composition operators on $H^{2}$, Indiana Univ. Math. J. 23 (1973/74), 471-496. MR 48:4816

11. J. H. Shapiro, Composition Operators and Classical Function Theory, Springer-Verlag, 1993. MR 94k:47049

12. J. H. Shapiro, The essential norm of a composition operator, Annals of Math. 125 (1987), 375-404. MR 88c: 47058

13. D. Stegenga and K. Stephenson, A geometric characterization of analytic functions with bounded mean oscillation, J. London Math. Soc. (2) 24 (1981), 243-254. MR 82m:30036

Department of Mathematics, Box 354350, University of Washington, Seattle, WashINGTON 98195-4350

E-mail address: poggi@math.washington.edu 